\title{
Opinión de los pacientes tras un programa de ejercicio físico domiciliario
}

\author{
Anna Junqué1, Yolanda Molina ${ }^{1}$, Yolanda Fernández², Lola Andreu³, Eva Segura ${ }^{4}$ \\ ${ }^{1}$ DUE Nefrologia, Hospital de Terrassa. Consorci Sanitari de Terrassa. Barcelona. España \\ ${ }^{2}$ Coordinadora de Hemodiálisis. Hospital de Terrassa. Consorci Sanitari de Terrassa. Barcelona. España \\ ${ }^{3}$ Departament d'Infermeria Fonamental i Medicoquirúrgica. Facultat de Medicina i Ciències de la Salut. Universi- \\ tat de Barcelona. Barcelona. España \\ ${ }^{4}$ Departament de Fisioteràpia. Universidad Cardenal Herrera-CEU, CEU Universities. València. España
}

\begin{abstract}
Como citar este artículo:
Junqué A, Molina Y, Fernández Y, Andreu L, Segura E. Opinión de los pacientes tras un programa de ejercicio físico domiciliario.

Enferm Nefrol. 2019 0ct-Dic;22(4):406-414
\end{abstract}

\section{Resumen}

Introducción. Las personas con enfermedad renal deben recibir una atención integral que incluya programas de ejercicio físico adaptado a sus necesidades.

Objetivo. Evaluar la satisfacción de los pacientes con enfermedad renal crónica en estadios IV y $\mathrm{V}$ ante un programa de ejercicio físico domiciliario.

Material y Método. Estudio descriptivo transversal en pacientes que realizaron un programa completo de entrenamiento físico domiciliario de 12 semanas de duración. Tras esta intervención, respondieron de forma anónima a un cuestionario ad-hoc validado por expertos, sobre su opinión acerca del programa.

Resultados. Participaron 62 pacientes. 24 estaban en programa de hemodiálisis, 17 en diálisis peritoneal y 7 en situación de enfermedad renal crónica estadio IV. 34 eran hombres. La edad media fue de $67,4 \pm 14,9$ años. 52 pacientes realizaron el programa solos en su domicilio. 33 de los pacientes les pareció muy correcto que el programa fuera domiciliario, 15 correcto y 2 poco correcto. 47 de los participantes consideraron muy correcto que la persona que dirigiera el programa fuera una enfermera conocida. 19 consideró que tras el

\section{Correspondencia:}

Anna Junqué Jiménez E-mail: annajunque@yahoo.es programa habían mejorado mucho, 14 que habían mejorado, 9 que habían mejorado poco y 3 que no habían mejorado. 39 estuvieron muy satisfechos de haber podido participar en el programa, 6 satisfechos, 1 poco satisfecho y 1 de los pacientes no estuvo satisfecho. No hubo diferencias significativas en las respuestas en relación al sexo, edad, tipo de tratamiento, o realizar el programa solo o acompañado.

Conclusiones. El programa de ejercicio físico domiciliario fue bien valorado por los pacientes que en su mayoría manifestaron haber mejorado y consideraron adecuado que fuera una enfermera la que dirigiera el programa.

PALABRAS CLAVE. satisfacción del paciente; ejercicio físico; enfermedad renal crónica.

\section{Patient opinion after a home physical exercise program}

\section{Abstract}

Objective. To evaluate patient satisfaction chronic kidney disease in stages IV and V after a home physical exercise program.

Material and methods. Descriptive cross-sectional study in patients who carried out a complete 12-week home physical training program. After this intervention, 
they responded anonymously to an ad-hoc questionnaire validated by experts, about their opinion about the program.

Results. 62 patients participated. 24 were on a hemodialysis program, 17 on peritoneal dialysis and 7 on stage IV chronic kidney disease. 34 were men. The mean age was $67.4 \pm 14.9$ years. 52 patients carried out the program alone at home. 33 of the patients considered as very correct that the program was domiciliary, 15 correct and 2 not correct. 47 of the participants considered as very correct that the person leading the program was a well-known nurse. 19 responded that after the program the improvement was a lot, 14 a good improvement, 9 little improvement and 3 that had not improved. 39 were very satisfied to participate in the program, 6 satisfied, 1 not very satisfied and 1 of the patients was not satisfied. There were no significant differences in the answers in relation to sex, age, type of treatment, or if the program was done alone or accompanied.

Conclusions. The home physical exercise program was well valued by the patients, who mostly said they had improved and considered as appropriate that a nurse leads the program.

Keywords. patient satisfaction; physical exercise; chronic kidney disease.

\section{Introducción}

La enfermedad renal crónica (ERC) es un importante problema emergente en todo el mundo; se relaciona primordialmente con el envejecimiento de la población, pero también con hábitos de vida poco saludables y factores de riesgo como la obesidad o problemas cardiovasculares. Las personas con ERC además de presentar una elevada mortalidad y numerosas patologías asociadas, muestran una disminución de su condición física y una deteriorada calidad de vida relacionada con la salud ${ }^{1,2}$, se caracterizan por ser mayoritariamente personas de edad avanzada, con neuropatía y miopatía urémica, catabolismo proteico alterado, anemia y son, en general, personas muy sedentarias, con una importante limitación en las actividades de la vida diaria. Por estos factores en las guías médicas nefrológicas se recomienda que los pacientes debieran realizar una adecuada rehabilitación física para poder enlentecer su deterioro muscular, preservar la capacidad funcional y su autonomía ${ }^{3-5}$.
Existe en la literatura la evidencia de los beneficios del ejercicio físico en pacientes renales, desde principios de la década de 1980 se empezaron a implementar programas de ejercicio físico (PEF) durante las sesiones de hemodiálisis como parte del tratamiento del paciente renal para mejorar la fuerza muscular, capacidad funcional y la calidad de vida relacionada con la salud de estas personas ${ }^{6-11}$. En estos últimos años, en España, algunas publicaciones avalan estos programas de ejercicio físico, pero su implementación sigue siendo muy lenta y dificultosa ${ }^{12-16}$. La falta de implantación es un problema que requiere fuerza política para resaltar la difícil situación de las personas con enfermedad renal, y se debiera alentar a los financiadores y proveedores a desarrollar modelos de atención íntegros, con PEF para minimizar la disminución de la condición física de estos pacientes. Existen responsables de servicios de nefrología que están comprometidos con estos programas pero generalmente son una pequeña excepción ${ }^{17}$. Recientemente, organizaciones internacionales como la European Association of Rehabilitation in Chronic Kidney Disease (EURORECKD) y el Global Renal Exercise group (G-REX) han estado desarrollando redes y recursos para la mejorar de los pacientes renales a través del ejercicio físico con un objetivo claro: mantener su calidad de vida y no solo limpiar sangre ${ }^{17}$.

Independientemente de la financiación de los PEF, una de las barreras más importantes es la de la motivación, tanto por parte del paciente como del personal sanitario. La motivación del personal sanitario puede verse afectada por la logística y las estructuras de las unidades de nefrología, las cuales están poco preparadas para implementar PEF, entre otros motivos por la sobrecarga de trabajo en la atención a pacientes crónicos complejos, de avanzada edad y comorbilidad asociada; además de plantillas escasas y sin formación en programas de ejercicio físico, lo que puede afectar al estrés del personal de nefrología, siendo todo ello un hándicap importante para poner en marcha programas específi$\cos$ de ejercicio del paciente renal ${ }^{18,19}$.

La barrera motivacional por parte del paciente también está mermada, ya que a lo largo del proceso de la enfermedad, el paciente se va deteriorando físicamente y mentalmente, y cuando llega a etapas tardías de la enfermedad, ya se ha producido una pérdida importante de la función renal y ha perdido la motivación para la realización de ejercicio físico, y por lo tanto, es habitual escuchar a los profesionales sanitarios comentar que los pacientes son demasiado frágiles o desmotivados. Sin 
embargo, existen artículos en la actualidad, que afirman que todos los pacientes renales, independientemente, de su fragilidad pueden participar en estos PEF ${ }^{20,21}$.

Los profesionales del ejercicio, rehabilitadores o fisioterapeutas, con un gran conocimiento del tema, podrían ayudar con la implantación y desarrollo de los programas en las unidades de nefrología, tanto para la evaluación de la capacidad funcional y el estado físico del paciente como en la programación de la prescripción de PEF individualizados. Sin embargo, es una barrera importante que se encuentran en la mayoría de unidades y es irrazonable esperar que un solo profesional pueda implementar y sostener un PEF en todos los estadios de la enfermedad y en todas las modalidades de tratamiento en el caso del estadio $\mathrm{V}^{22}$.

Ante estas limitaciones y con el fin de promover la autonomía del paciente, existe la opción de pautar un programa de ejercicio en su propio domicilio. Algunos estudios han comparado los efectos de un programa de ejercicio físico intradiálisis frente a uno domiciliario, con resultados muy positivos ${ }^{18-20}$.

Por otra parte, no siempre se conoce el grado de satisfacción o la opinión de los pacientes respecto a los $P E F$, dicha opinión es una fuente de información imprescindible para implementar cualquier estrategia de mejora y puede ser la clave para lograr una máxima adherencia al tratamiento y ayudar a divulgar entre los pacientes la importancia y el papel beneficioso del ejercicio frente al deterioro físico que sufren las personas con enfermedad renal $22,23$.

Conociendo la importancia de que las personas con ERC reciban una atención integral y conocer su opinión, nos plantemos como objetivo evaluar la satisfacción de los pacientes con enfermedad renal crónica en estadios IV y $\mathrm{V}$ ante un programa de ejercicio físico domiciliario, así como identificar posibles áreas de mejora.

\section{Material y Método}

Se realizó un estudio descriptivo transversal para evaluar la satisfacción de un programa de ejercicio físico domiciliario desarrollado a partir de un estudio prospectivo de 12 semanas de duración a pacientes con ERC, en estadio IV-V, HD y DP, tratados en el Hospital de Terrassa y que fue aprobado por su comité ético y realizado de acuerdo con las indicaciones y los requerimientos expresados en las normas internacionales rela- tivas a la realización de estudios de la Declaración de Helsinki (revisión de Seúl, octubre 2008).

Como criterios de inclusión se establecieron: el consentimiento informado, edad igual o superior a 18 años, llevar más de tres meses en el centro. Los criterios de exclusión fueron: la imposibilidad física para realizar el programa de ejercicio físico, la no aceptación en la participación del estudio, padecer alguna enfermedad infecciosa potencialmente transmisible y estar participando activamente en algún otro proyecto que pudiera influir en la condición física y en el nivel de actividad física del paciente.

La muestra del estudio fue no probabilística y la técnica de muestreo fue de conveniencia ya que seleccionamos a los sujetos que cumplían los criterios de inclusión y formaban parte de nuestro centro hospitalario y se visitaron durante un período de tiempo de medio año establecido previamente.

Los pacientes reclutados se sometieron a un programa de ejercicio físico domiciliario (PEFD), similar al diseñado en un estudio anterior en el que se comparaba los efectos del programa al aplicarlo intradialisis, guiado por el personal de enfermería de la unidad, o realizado en el domicilio supervisado por un fisioterapeuta ${ }^{16}$.

En el PEFD se combinaban ejercicios de fuerza, aeróbicos y de equilibrio estructurados en series de deambulación y musculación que se repetían, aumentando progresivamente su duración e intensidad según la capacidad de cada individuo. Se instruyó a los pacientes para realizar 3 sesiones semanales de aproximadamente 45 minutos en días de elección propia (a los pacientes en HD, se aconsejaba en el día libre de diálisis).

Para hacer un seguimiento de la cumplimentación del PEFD los participantes disponían de un diario de registro de la actividad donde debían anotar cada día las series, repeticiones de cada ejercicio, si utilizaban un peso lastrado, y además registraban sus tensiones arteriales y frecuencia cardíaca antes y después de cada sesión. En el mismo diario de registro, los participantes tenían una guía con explicación e imágenes de cada ejercicio.

Para poder controlar su evolución o solventar cualquier duda o problema, los participantes disponían de un teléfono directo con la enfermera responsable del estudio de lunes a viernes de 8 a 17h. A su vez, dicha enfermera realizaba una llamada semanal para resumir el trabajo realizado. 
Para fomentar la adherencia al PEFD se utilizó, además del seguimiento de la enfermera responsable, un método de gamificación, técnica de aprendizaje que traslada la mecánica de los juegos al ámbito educativo-profesional. En nuestro estudio, para estimular a los pacientes, diseñamos un póster que se colocó en la pared de entrada al servicio de nefrología. Este póster constaba de tres columnas que mostraban de forma anónima el nivel de progresión de cada paciente. De esta manera, el paciente cuando acudía al servicio de nefrología, veía su progresión y podía sentirse estimulado para seguir y mejorar frente a los demás participantes del estudio (Figura 1).

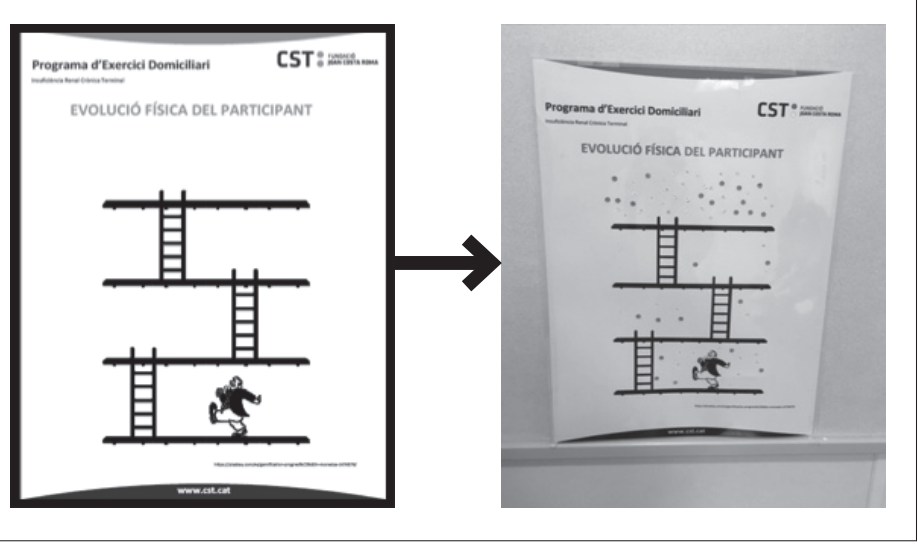

Figura 1. Gamificación para los participantes al estudio.

Al finalizar las 12 semanas del PEFD, se entregó al participante un cuestionario anónimo ad-hoc, validado por expertos, para conocer su grado de satisfacción. En este cuestionario se incluyó una introducción con las instrucciones para responder a las preguntas, una información básica sobre edad, sexo, modalidad de tratamiento y si realizaba el programa de ejercicios solo o acompañado. A continuación, 6 preguntas con una escala psicométrica, tipo Likert, con 4 posibles respuestas, de 1, nada satisfecho a 4, muy satisfecho. Las preguntas fueron:

1. ¿Le ha parecido correcto que el programa tenga que realizarse en casa, tres días a la semana, durante 45 minutos?

2. ¿El material que se le ha entregado ha sido comprensible y fácil de registrar? el porcentaje.
3. ¿Está de acuerdo en que el programa es seguro y fácil de realizar?

4. ¿El profesional que le ha atendido ha sido correcto y le ha sabido explicar el programa de ejercicios?

5. Tras los ejercicios realizados, ¿considera usted que tiene más fuerza muscular que al inicio?

6. ¿Está usted satisfecho de haber participado y de todos los controles que se la han realizado?

El análisis estadístico de las variables recogidas se analizaron con el programa SPSS versión 24 (SPSS INC, Chicago, IL, EEUU). Las variables continuas se describieron con la media y la desviación estándar y las variables cualitativas se expresaron con el porcentaje. Para la comparación de las variables se utilizó la prueba del Chi cuadrado para variables categóricas mediante tablas de contingencia. Los resultados se consideraron estadísticamente significativos si $p$ valor fue inferior a 0,05 .

\section{Resultados}

Participaron en el estudio 62 pacientes (50\% HD, $36 \%$ DP y $14 \%$ ERC estadio IV), $69 \%$ hombres y un $31 \%$ mujeres. La media de edad de la muestra fue de $67,4 \pm 14,9$. En la Tabla 1 se muestran los principales datos demográficos y etiologías de la enfermedad renal. 52 pacientes realizaron los programas solos y 10 afirmaron que lo realizaban acompañados de un familiar. 33 de los pacientes les pareció muy correcto que el programa fuera domiciliario, 15 correcto y 2 de los participantes les pareció poco correcto. 35 de los

Tabla 1. Datos demográficos, comorbilidad y etiología.

\begin{tabular}{|l|l|l|l|l|}
\hline & Total & HD & DP & ERCA \\
\hline Edad (años) & $67,4 \pm 14,9$ & $68,0 \pm 15,0$ & $68,2 \pm 15,2$ & $63,8 \pm 14,9$ \\
\hline Tiempo en TSR (meses) & $40,9 \pm 66,2$ & $47,3 \pm 73,8$ & $28,6 \pm 47,6$ & ---- \\
\hline Sexo (\%hombres) & $60,4 \%$ & $60 \%$ & $61 \%$ & $70 \%$ \\
\hline Índice de Charlson & $8,2 \pm 2,6$ & $8,5 \pm 2,5$ & $7,6 \pm 2,5$ & $7,5 \pm 2,5$ \\
\hline Glomerulonefritis (\%) & $13,2 \%$ & $14,3 \%$ & $14,3 \%$ & $14 \%$ \\
\hline Hipertensión (\%) & $11,3 \%$ & $17,1 \%$ & $17,1 \%$ & $15 \%$ \\
\hline Diabetes Mellitus (\%) & $17,0 \%$ & $17,1 \%$ & $17,1 \%$ & $18 \%$ \\
\hline
\end{tabular}

Las variables continuas se describen con la media y la desviación estándar y las variables cualitativas se expresan con

HD: hemodiálisis, DP: diálisis peritoneal, ERCA: enfermedad renal crónica avanzada. $\mathrm{N}=62(50 \%$ HD, 36\% DP $14 \%$ ERCA). 
participantes les pareció muy fácil y seguro, 11 fácil y seguro y 4 poco seguro y difícil. 47 de los participantes encontraron muy correcto que la persona que lideraba el programa fuera una enfermera renal conocida por ellos, 2 correcto y 1 participante le pareció poco correcto. 19 consideraron que habían mejorado mucho, 14 que habían mejorado, 9 que habían mejorado poco y 3 que no habían mejorado. 39 estuvo muy satisfecho de haber podido participar en el programa, 6 satisfecho, 1 poco satisfecho y 1 no estuvo satisfecho. La Figura 2 muestra los resultados de todas las preguntas y la Tabla 2 refleja los resultados de las respuestas del cuestionario según la modalidad de tratamiento a que se sometía el paciente. Finalmente, en la Tabla 3 podemos observar los resultados del cuestionario dependiendo de la edad, mayores o menores de 70 años, un $42,9 \%$ eran menores de 70 años y un 57,1\% mayores de 70 años. En esta tabla observamos que no hay diferencias significativas en base a la edad y las respuestas de las 6 peguntas del cuestionario, sin embargo, debemos mencionar que la pregunta 2 , casi alcanza la significación estadística $(p=0,051)$.

\section{Discusión}

Con mayor frecuencia la evidencia muestra que los PEF en pacientes renales mejoran su condición física y su calidad de vida relacionada con la salud ${ }^{14,15}$. En nuestro estudio el PEFD, además de ser efectivo en cuanto a la mejoría de la fuerza muscular, generan en los pacientes satisfacción, esto implica ofrecer buenos cuidados, pues es conocido que la satisfacción siempre está vinculada a las expectativas de los pacientes en relación a los cuidados de salud recibidos ${ }^{16,24}$.

No hemos encontrado ningún estudio que analice exclusivamente la satisfacción de los programas de ejercicio físico en pacientes renales, aunque un artículo de 2002, comparan un programa de ejercicio intradiálisis con uno domiciliario y aunque los resultados del estudio eran positivos, los pacientes afirmaban preferir el ejercicio intradiálisis ${ }^{25}$. Nosotros también pensamos que ha sido beneficioso para los pacientes que se realizara el programa en casa al permitirles hacer la actividad más libremente, y cabe destacar que un $21 \%$ de los pacientes llevaron a cabo el PEFD acompañados, lo que sin duda reforzó sus vínculos familiares.

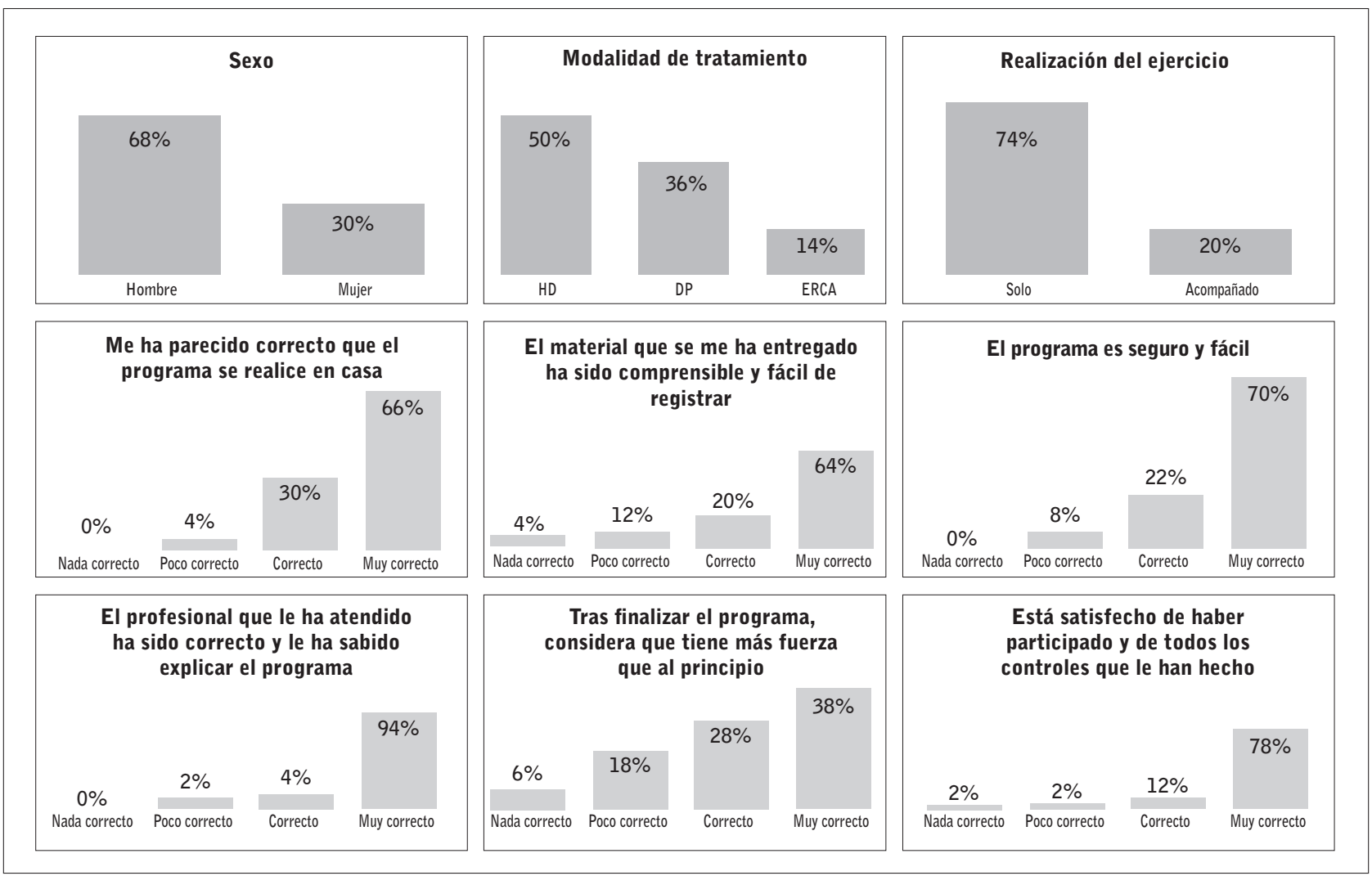

Figura 2. Gráficos con los resultados del cuestionario de satisfacción. 
Tabla 2. Resultados de las preguntas del cuestionario según la modalidad de tratamiento o etapa de la enfermedad renal.

\begin{tabular}{|c|c|c|c|c|c|c|c|c|c|c|c|c|}
\hline \multirow[b]{2}{*}{ Puntuaciones } & \multicolumn{4}{|c|}{ HD } & \multicolumn{4}{|c|}{ DP } & \multicolumn{4}{|c|}{ ERCA } \\
\hline & 1 & 2 & 3 & 4 & 1 & 2 & 3 & 4 & 1 & 2 & 3 & 4 \\
\hline Pregunta 1 & 0 & 1 & 8 & 15 & 0 & 1 & 4 & 13 & 0 & 0 & 3 & 4 \\
\hline Pregunta 2 & 1 & 0 & 5 & 18 & 0 & 6 & 2 & 10 & 1 & 0 & 2 & 4 \\
\hline Pregunta 3 & 0 & 1 & 8 & 15 & 0 & 2 & 2 & 14 & 0 & 1 & 1 & 5 \\
\hline Pregunta 4 & 1 & 0 & 1 & 22 & 0 & 0 & 1 & 17 & 0 & 0 & 0 & 7 \\
\hline Pregunta 5 & 2 & 3 & 9 & 9 & 0 & 5 & 3 & 7 & 1 & 1 & 1 & 3 \\
\hline Pregunta 6 & 1 & 0 & 5 & 17 & 0 & 1 & 1 & 14 & 0 & 0 & 0 & 7 \\
\hline
\end{tabular}

1 nada satisfecho, 2 poco satisfecho, 3 satisfecho, 4 muy satisfecho.

Por otra parte, existen algunas publicaciones donde coinciden en que los pacientes que participan en programas de ejercicio domiciliario, están satisfechos en que el programa sea liderado por la figura de una enfermera de nefrología con soporte de otros profesionales ${ }^{26}$. Estos resultados son semejantes a los nuestros ya que

Tabla 3. Resultado de las preguntas dependiendo de la edad.

\begin{tabular}{|c|c|c|c|c|}
\hline Preguntas & Respuestas & $\begin{array}{l}\text { Participantes } \\
\leq 70 \text { años }\end{array}$ & $\begin{array}{l}\text { Participantes } \\
\geq 70 \text { años }\end{array}$ & $\mathbf{p}$ \\
\hline \multirow[t]{4}{*}{ Pregunta 1} & 1 & 0 & 0 & \multirow[t]{4}{*}{0,314} \\
\hline & 2 & 1 & 1 & \\
\hline & 3 & 4 & 11 & \\
\hline & 4 & 16 & 16 & \\
\hline \multirow[t]{4}{*}{ Pregunta 2} & 1 & 0 & 2 & \multirow[t]{4}{*}{0,051} \\
\hline & 2 & 2 & 4 & \\
\hline & 3 & 1 & 8 & \\
\hline & 4 & 18 & 14 & \\
\hline \multirow[t]{4}{*}{ Pregunta 3} & 1 & 0 & 0 & \multirow[t]{4}{*}{0,064} \\
\hline & 2 & 0 & 4 & \\
\hline & 3 & 3 & 8 & \\
\hline & 4 & 18 & 16 & \\
\hline \multirow[t]{4}{*}{ Pregunta 4} & 1 & 0 & 1 & \multirow[t]{4}{*}{0,671} \\
\hline & 2 & 0 & 0 & \\
\hline & 3 & 1 & 1 & \\
\hline & 4 & 20 & 26 & \\
\hline \multirow[t]{4}{*}{ Pregunta 5} & 1 & 1 & 2 & \multirow[t]{4}{*}{0,159} \\
\hline & 2 & 1 & 8 & \\
\hline & 3 & 7 & 6 & \\
\hline & 4 & 10 & 9 & \\
\hline \multirow[t]{4}{*}{ Pregunta 6} & 1 & 0 & 1 & \multirow[t]{4}{*}{0,532} \\
\hline & 2 & 1 & 0 & \\
\hline & 3 & 3 & 3 & \\
\hline & 4 & 16 & 22 & \\
\hline
\end{tabular}

Participantes $\leq 70$ años $42,9 \%$. Participantes $\geq 70$ años $57,1 \%$ 1 nada satisfecho, 2 poco satisfecho, 3 satisfecho, 4 muy satisfecho Significación estadísitca * $p \leq 0,05$. el $94 \%$ de los participantes les pareció muy correcto que liderara el programa una enfermera experta en el cuidado renal.

En nuestro estudio, al igual que en otros encontrados en la literatura, la enfermera que atiende al paciente renal ha de motivar a los pacientes e incentivarlos para que tengan una adecuada adherencia terapéutica ${ }^{27,28}$. En nuestro caso, la enfermera realizaba una llamada semanal y en el servicio de nefrología se utilizaba como un póster basado en gamificación para estimular a los participantes, lo que parece resulto efectivo pues pocos pacientes no estuvieron de acuerdo con alguna parte del programa.

Existen diversos tipos de programas domiciliarios publicados: un programa de yoga con muy buena adherencia y muy buenos resultados ${ }^{29}$, un programa de Tai $\mathrm{Chi}^{30}$ y más recientemente programas aeróbicos y otros de fuerza resistencia o combinados $^{31}$. Nuestro programa, intercala ejercicios aeróbicos, anaeróbicos y de equilibrio, es decir, creemos que es un programa muy completo y los resultados nos muestran que el $92 \%$ de nuestros los pacientes lo encuentran fácil y seguro.

Nuestros resultados son similares a los mostrados en la literatura $26,31,32$, puesto que el $73 \%$ de los pacientes afirman que ha mejorado su condición física y un $83 \%$ afirman estar muy satisfechos y un $13 \%$ satisfechos. Debemos comentar que el cuestionario se pasó a todos los participantes del estudio por lo que no se tuvo en cuenta el grado de adherencia al programa, por eso aventuramos, ya que las encuestas eran anónimas, que las personas que manifestaban no estar satisfechas y no haber tenido mejoras era porque tampoco habían seguido adecuadamente el programa de ejercicios. 
En nuestro, estudio podemos observar que la satisfacción por parte de los participantes es independiente de otras características como la edad y solamente casi alcanza la significación estadística $(p=0,051$ ), la pregunta 2 , ( ¿el material es comprensible y fácil de registrar?), en la que hay diferencias entre las respuestas de los más jóvenes y los de edad avanzada, por esto consideramos que es necesario utilizar imágenes y registros muy asequibles pensando en las personas de edad, que frecuentemente tienen déficits visuales y les tiembla el pulso. Sin embargo, independientemente de este resultado podemos constatar que en nuestro estudio los PEFD pueden ser divulgados y practicados por todo tipo de persona con ERC independientemente de la edad y modalidad de tratamiento.

Como conclusión, se puede afirmar que los pacientes han estado muy satisfechos con el programa de ejercicio físico domiciliario y manifiestan haber mejorado. Lo han encontrado seguro y fácil y les ha parecido muy correcto que lo lidere una enfermera de nefrología. Estos programas pueden ser beneficiosos para todos los pacientes con ERC, independientemente del estadio de la enfermedad o modalidad de tratamiento que reciban y puede ser una alternativa terapéutica para aquellos servicios de nefrología que no disponen de infraestructura, organización y los recursos humanos necesarios para realizar los programas en el mismo servicio de nefrología.

\section{Agradecimientos}

A todos los pacientes por su valiosa colaboración en la presente investigación.

Este trabajo de investigación se ha realizado en el marco de programa de Doctorado en Enfermería y Salud de la Universidad de Barcelona (UB).

Recibido: 30-07-19

Revisado: 17-08-19

Modificado: 19-08-19

Aceptado: 22-08-19

\section{Bibliografía}

1. Otero A, de Francisco A, Gayoso P, García F, EPIRCE Study Group. Prevalence of chronic renal disease in Spain: results of the EPIRCE study. $\mathrm{Ne}$ frologia. 2010;30(1):78-86.

2. Fassbinder OB de Tânia Regina Cavinatto, Winkelmann, Eliane Roseli, Schneider, Juliana, Wendland, Juliana, Oliveira. Capacidade funcional e qualidade de vida de pacientes com doença renal crônica pré-dialítica e em hemodiálise - Um estudo transversal. Braz J Nephrol. 2015;37:47-54.

3. Nelson RG, Tuttle KR. The new KDOQI clinical practice guidelines and clinical practice recommendations for diabetes and CKD. Blodd Purif. 2007;25(1):112-4.

4. Smart NA, Williams AD, Levinger I, Selig S, Howden E, Coomes JS, et al. Exercise \& Sports Science Australia (ESSA) position statement on exercise and chronic kidney disease. J Sci Med Sport. 2013;16(5):406-11.

5. Nesrallah GE, Mustafa RA, MacRae J, Pauly RP, Perkins DN, Gangji A et al. Canadian Society of Nephrology guidelines for the management of patients with ESRD treated with intensive hemodialysis. Am J Kidney Dis. 2013;62(1):187-98.

6. Segura-Ortí E, Kouidi E, Lisón JF. Effect of resistance exercise during hemodialysis on physical function and quality of life: randomized controlled trial. Clin Nephrol. 1 de mayo de 2009;71(05):52737.

7. Heiwe S, Jacobson SH. Exercise training for adults with chronic kidney disease. Cochrane Database Syst Rev. 5 de octubre de 2011;(10):CD003236.

8. Oh-Park M, Fast A, Gopal S, Lynn R, Frei G, Drenth $R$, et al. Exercise for the dialyzed: aerobic and strength training during hemodialysis. Am J Phys Med Rehabil. noviembre de 2002;81(11):81421.

9. Mauricia Cristina de Lima. Effect of Exercise Performed during Hemodialysis: Strength versus Aerobic. Ren Fail. 2013;35(5):697-704. 
10. Segura-Ortí E. [Exercise in haemodyalisis patients: a literature systematic review]. Nefrol Publicacion of Soc Espanola Nefrol. 2010;30(2):236-46.

11. DePaul V, Moreland J, Eager T, Clase CM. The effectiveness of aerobic and muscle strength training in patients receiving hemodialysis and EPO: a randomized controlled trial. Am J Kidney Dis Off $\mathrm{J}$ Natl Kidney Found. diciembre de 2002;40(6):1219-29.

12. Lucia Ortega. Comparation of two exercise programs for hemodialysis patients, intradialysis vs home based program. Absolute and relative reliability of physical performance. [Valencia]: Universidad Cardenal Herrera.

13. Esteve Simo M Vicent, Junqué Jiménez, Anna, Moreno Guzmán, Fátima, Carneiro Oliveira, José, Fulquet Nicolas, Miquel, Pou Potau, Mónica, Saurina Sole, Anna, Duarte Gallego, Verónica, Tapia Gonzalez, Irati, Ramirez de Arellano. Beneficios del ejercicio físico de baja intensidad durante la sesión de hemodiálisis en el paciente anciano. Nefrol Madr. 2015;35:385-94.

14. Esteve Simó $V$, Junqué $A$, Fulquet $M$, Duarte $V$, Saurina A, Pou M, et al. Complete Low-Intensity Endurance Training Programme in Haemodialysis Patients: Improving the Care of Renal Patients. Nephron Clin Pract. 17 de diciembre de 2014;128(3-4):387-93.

15. Junqué Jiménez $A$, Esteve Simó $V$, Tomás Bernaveu E, Paz López Ó, Iza Pinedo G, Luceño Solé I, et al. Effects of an adapted physical activity program in elderly haemodialysis patients. Enferm Nefrológica. enero de $2015 ; 18(1): 11-8$.

16. Pérez Dominguez FB. Comparación de los efectos de un programa de ejercicio intradiálisis frente a un programa de ejercicio domiciliario. [Valencia]: Universidad Cardenal Herrera; 2016.

17. Bennett PN, Thompson S, Wilund KR. An introduction to Exercise and Physical Activity in Dialysis Patients: Preventing the unacceptable journey to physical dysfunction. Semin Dial. 2019;32(4):281-2.

18. Darawad MW, Khalil AA. Jordanian Dialysis Patients' Perceived Exercise Benefits and Barriers: A correlation study. Rehabil Nurs. 2013;38(6):31522.
19. Zelle DM, Corpeleijn E, Klaassen G, Schutte $E_{\text {, }}$ Navis G, Bakker SJL. Fear of Movement and Low Self-Efficacy Are Important Barriers in Physical Activity after Renal Transplantation. PLoS ONE [Internet]. Febrero 2016 [Consultado 21 oct 2019];11(2). Disponible en: https://www.ncbi.nlm. nih.gov/pmc/articles/PMC4742485/.

20. Chu NM, McAdams-DeMarco MA. Exercise and cognitive function in patients with end-stage kidney disease. Semin Dial. 2019;32(4):283-90.

21. Wang CJ, Johansen KL. Are dialysis patients too frail to exercise? Semin Dial. 2019;32(4):291-6.

22. Clarke $A L$, Jhamb $M$, Bennett $P N$. Barriers and facilitators for engagement and implementation of exercise in end-stage kidney disease: Future theory-based interventions using the Behavior Change Wheel. Semin Dial. 2019;32(4):308-19.

23. Roshanravan B, Patel KV. Assessment of physical functioning in the clinical care of the patient with advanced kidney disease. Semin Dial. 2019;32(4):351-60.

24. Baria $F$, Kamimura MA, Aoike DT, Ammirati $A$, Rocha ML, de Mello MT, et al. Randomized controlled trial to evaluate the impact of aerobic exercise on visceral fat in overweight chronic kidney disease patients. Nephrol Dial Transplant Off Publ Eur Dial Transpl Assoc - Eur Ren Assoc. abril de 2014;29(4):857-64.

25. Konstantinidou E, Koukouvou G, Kouidi E, Deligiannis $A$, Tourkantonis $A$. Exercise training in patients with end-stage renal disease on hemodialysis: comparison of three rehabilitation programs. J Rehabil Med Taylor Francis Ltd. enero de 2002;34(1):40-5.

26. Tao X, Chow SKY, Wong FK. The effects of a nurse-supervised home exercise programme on improving patients' perceptions of the benefits and barriers to exercise: A randomised controlled trial. J Clin Nurs. septiembre de 2017;26(17-18):276575.

27. Tao $X$, Chow SKY, Wong FKY. A nurse-led case management program on home exercise training for hemodialysis patients: A randomized controlled trial. Int J Nurs Stud. junio de 2015;52(6):102941. 
28. Fournier J. Nurse-led home exercise programme improves physical function for people receiving haemodialysis. Evid Based Nurs. enero de 2016;19(1):12.

29. Yurtkuran M, Alp A, Yurtkuran M, Dilek K. A modified yoga-based exercise program in hemodialysis patients: a randomized controlled study. Complement Ther Med. septiembre de 2007;15(3):164-71.

30. Ling K, Wong FSY, Chan W, Chan S, Chan EPY, Cheng $Y$, et al. Effect of a home exercise program based on tai chi in patients with end-stage renal disease. Perit Dial Int J Int Soc Perit Dial. diciembre de 2003;23(Supl 2):S99-103.

31. Manfredini F, Mallamaci F, D'Arrigo G, Baggetta R, Bolignano $D$, Torino $C$, et al. Exercise in Patients on Dialysis: A Multicenter, Randomized Clinical Trial. J Am Soc Nephrol JASN. 2017;28(4):1259-68.

32. Hiraki K, Shibagaki Y, Izawa KP, Hotta C, Wakamiya A, Sakurada T, et al. Effects of home-based exercise on pre-dialysis chronic kidney disease patients: a randomized pilot and feasibility trial. BMC Nephrol. 17 de junio de 2017;18(1):198. Rev. Esp. Salud Pública. 2010;84(2):169-84.

Este artículo se distribuye bajo una Licencia Creative Commons Atribución-NoComercial 4.0 Internacional. https://creativecommons.org/licenses/by-nc/4.0/

Open Access (C) (;) (8) 